\title{
NIR responsive liposomal system for rapid release of drugs in cancer therapy
}

\author{
This article was published in the following Dove Press journal: \\ International Journal of Nanomedicine \\ 6 June 2017 \\ Number of times this article has been viewed
}

\author{
Ming-Mao Chen' \\ Yuan-Yuan Liu' \\ Guang-Hao Su ${ }^{2}$ \\ Fei-Fei Song' \\ Yan $\mathrm{Liu}^{3}$ \\ Qi-Qing Zhang',4
}

'Institute of Biomedical and Pharmaceutical Technology, Fuzhou

University, Fuzhou, ${ }^{2}$ Institute of

Pediatric Research, Children's

Hospital of Soochow University,

Suzhou, ${ }^{3}$ State Key Lab of Structural

Chemistry, Fujian Institute of

Research on the Structure of Matter,

Chinese Academy of Sciences, Fuzhou,

${ }^{4}$ Key Laboratory of Biomedical

Material of Tianjin, Institute of

Biomedical Engineering, Chinese

Academy of Medical Science \& Peking

Union Medical College, Tianjin,

People's Republic of China
Correspondence: Yan Liu Fujian Institute of Research on the Structure of Matter, Chinese Academy of Sciences, I 55 Yangqiao Road West, Fuzhou, People's Republic of China

Tel +86 59I 63I7 94I6

Fax +86 591 6317 942I

Email liuyan@fjirsm.ac.cn

Qi-Qing Zhang

Institute of Biomedical and

Pharmaceutical Technology, Fuzhou

University, 523 Gongye Road, Fuzhou,

People's Republic of China

Tel/fax +86 59I 83725260

Email zhangqiq@।26.com

\begin{abstract}
To design a rapid release liposomal system for cancer therapy, a NIR responsive bubble-generating thermosensitive liposome (BTSL) system combined with photothermal agent (Cypate), doxorubicin (DOX), and $\mathrm{NH}_{4} \mathrm{HCO}_{3}$ was developed. Cypate/DOX-BTSL exhibited a good aqueous stability, photostability, and photothermal effect. In vitro release suggested that the amounts of DOX released from BTSL were obviously higher than that of $\left(\mathrm{NH}_{4}\right)_{2} \mathrm{SO}_{4}$ liposomes at $42^{\circ} \mathrm{C}$. After NIR irradiation, the hyperthermic temperature induced by Cypate led to the decomposition of $\mathrm{NH}_{4} \mathrm{HCO}_{3}$ and the generation of a large number of $\mathrm{CO}_{2}$ bubbles, triggering a rapid release of drugs. Confocal laser scanning microscope and acridine orange staining indicated that Cypate/DOX-BTSL upon irradiation could facilitate to disrupt the lysosomal membranes and realize endolysosomal escape into cytosol, improving the intracellular uptake of DOX clearly. MTT and trypan blue staining implied that the cell damage of Cypate/ DOX-BTSL with NIR irradiation was more severe than that in the groups without irradiation. In vivo results indicated that Cypate/DOX-BTSL with irradiation could dramatically increase the accumulation of DOX in tumor, inhibit tumor growth, and reduce systemic side effects of DOX. These data demonstrated that Cypate/DOX-BTSL has the potential to be used as a NIR responsive liposomal system for a rapid release of drugs in thermochemotherapy.
\end{abstract}

Keywords: NIR responsive, thermoresponsive liposome, triggered drug release, bubble-generating, thermochemotherapy

\section{Introduction}

Chemotherapy is still a major treatment modality for tumors, yet its efficacy is limited by the serious side effects on normal tissues. ${ }^{1-4}$ Photothermal therapy (PTT) has been considered as a noninvasive method of cancer therapy, which uses photothermal agents to generate heat under photoirradiation without injuring healthy tissues. ${ }^{5,6}$ Although PTT is highly effective in inhibiting tumor growth, it remains a major challenge to achieve tumor eradication without tumor regrowth. A combination therapy of PTT and chemotherapy has been considered as a promising strategy to improve therapeutic efficiency and minimize side effects. Nevertheless, to realize the maximal synergistic effect, the accurate doses of chemotherapy drug and photothermal agent should be simultaneously delivered to the same tumor cells.

Nanodrug delivery systems such as liposomes and nanoparticles have been developed to selectively accumulate in the tumor compartment via a passive targeting mechanism named the enhanced permeability and retention (EPR) effect, ${ }^{7,8}$ which can reduce potential toxicity and enhance effective therapy. PEGylated liposomes are recognized as stable vehicles of drugs; however, the liposomes need to be triggered to release the drugs after they are accumulated in the tumor cells for effective therapy. Thermosensitive liposomes can immediately be triggered for the rapid release of 
antitumor drugs by changing the permeability of liposomes at hyperthermic temperature $\left(40^{\circ} \mathrm{C}-42^{\circ} \mathrm{C}\right)$ in tumor sites. ${ }^{9,10}$ Recently, a bubble-generating thermosensitive liposome (BTSL) system that contained ammonium bicarbonate $\left(\mathrm{NH}_{4} \mathrm{HCO}_{3}\right)$ has been developed to facilitate the rapid release of drugs. The key to this liposome system is that $\mathrm{NH}_{4} \mathrm{HCO}_{3}$ can generate $\mathrm{CO}_{2}$ bubbles at a local temperature of $\sim 42^{\circ} \mathrm{C}$, and then the bubbles collapse to create permeable defects in the lipid bilayer and induce a rapid drug release. ${ }^{11,12}$ On the other hand, the lysosomal membranes are disrupted by the collapse of $\mathrm{CO}_{2}$ bubbles, which not only facilitate endolysosomal escape of drugs into cytosol but also release proteases into the cytosol to cause cell death. ${ }^{13-16}$

Although hyperthermia can induce the drugs to release from BTSL, photothermal effect from photothermal agents upon photoirradiation seems to be a better triggered release method. Under NIR irradiation, photothermal agents can raise the temperature to $42^{\circ} \mathrm{C}$ and induce the decomposition of $\mathrm{NH}_{4} \mathrm{HCO}_{3}$ to generate $\mathrm{CO}_{2}$ bubbles, which then cause the rapid drug release. Additionally, this novel BTSL system containing antitumor drugs and photothermal agents can enhance the synergistic effect of chemotherapy and PTT. Nevertheless, a study on the application of BTSL for the multimodal therapy has seldom been done so far.

Near-infrared organic cyanine dyes such as indocyanine green (ICG) and Cypate have been known as clinically potential theranostic agents, owing to their promising properties, including near-infrared fluorescence (NIRF) emission $>800 \mathrm{~nm}$, photoacoustic (PA) imaging, and PTT after excitation by near-infrared light. Cypate as a carboxylic derivative of ICG exhibits a better stability than ICG. ${ }^{17-22}$ Therefore, in this study, we chose Cypate as a model photothermal agent.

The aim of this work was to develop a NIR responsive liposomal system (Cypate/doxorubicin [DOX]-BTSL) for rapid release of drugs in synergistic therapy of PTT and chemotherapy. As shown in Figure 1, Cypate and DOX are encapsulated into the BTSL system containing $\mathrm{NH}_{4} \mathrm{HCO}_{3}$ first. At the tumor site, the liposomes suffer from NIR irradiation, which leads to a hyperthermic temperature and PTT. When the temperature is increased to $40^{\circ} \mathrm{C}-42^{\circ} \mathrm{C}$, the BTSLs generate $\mathrm{CO}_{2}$ bubbles due to the decomposition of $\mathrm{NH}_{4} \mathrm{HCO}_{3}$, inducing a rapid release of drugs, enhancing the synergistic therapeutic efficacy of chemotherapy and PTT. The physicochemical characteristics and release features of the liposomes were investigated to evaluate the potential to be used as a NIR responsive liposomal system for a rapid release of drugs. Moreover, photothermal effect, cellular uptake, tumor accumulation, and antitumor activity in vivo of the liposomes were assayed to demonstrate the effectiveness of this rapid drug release system for synergistic therapy of PTT and chemotherapy.

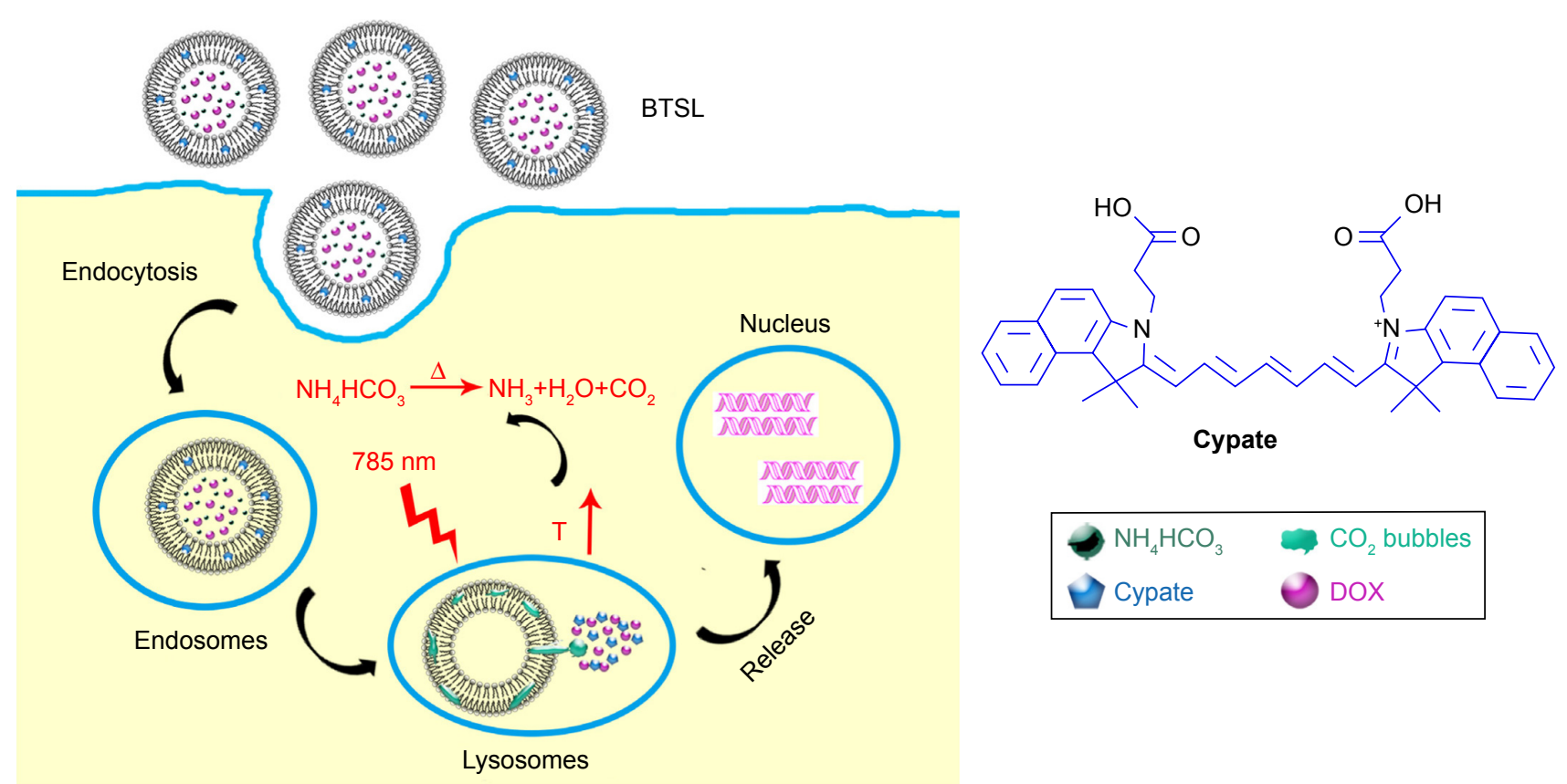

Figure I Schematic illustration of BTSL integrating Cypate dye and DOX for synergistic therapy of PTT and chemotherapy. Note: When the temperature is heated to $40^{\circ} \mathrm{C}-42^{\circ} \mathrm{C}$ due to NIR laser irradiation, the BTSL generate $\mathrm{CO}_{2}$ bubbles, inducing a rapid release of drugs. Abbreviations: BTSL, bubble-generating thermoresponsive liposome; DOX, doxorubicin; PTT, photothermal therapy. 


\section{Materials and methods Materials}

DOX (98\%) was purchased from Shanghai Civi Chemical Technology Co., Ltd. (Shanghai, People's Republic of China). Cypate was obtained from Fujian Institute of Research on Structure of Matter, Chinese Academy of Sciences (Fuzhou, People's Republic of China). RPMI 1640 medium was purchased from Hyclone (Logan, UT, USA). Dipalmitoylphosphatidylcholine (DPPC) and 1,2-distearylsn-glycero-3-phosphoethanaolamine-N-[methoxypoly(ethylene glycol)-2000] (DSPE-PEG ${ }_{2000}$ ) were obtained from Avanti Polar Lipids (Alabaster, AL, USA). Cholesterol (Chol), $\mathrm{NH}_{4} \mathrm{HCO}_{3}$, and $\left(\mathrm{NH}_{4}\right)_{2} \mathrm{SO}_{4}$ were supplied by Sinopharm Chemical Reagent Co., Ltd. (Shanghai, People's Republic of China). Human breast cancer (MCF-7) cells and mouse breast cancer (4T1) cells were purchased from the Cell Bank of Chinese Academy of Sciences (Shanghai, People's Republic of China). Other chemicals and reagents were of analytical grade.

\section{Preparation of liposomes}

Liposomes were prepared by thin-film hydration, followed by sonication method. ${ }^{23-26}$ In brief, DPPC, Chol, DSPE-PEG ${ }_{2000}$, and Cypate at a molar ratio of 12:8:1:0.8 were dissolved in a mixed solvent of chloroform and methanol $(\mathrm{V}: \mathrm{V}=1: 1)$ and placed in a high vacuum to remove the residual organic solvent. The lipid film was then hydrated using an aqueous $\left(\mathrm{NH}_{4}\right)_{2} \mathrm{SO}_{4}(350 \mathrm{mM})$ or $\mathrm{NH}_{4} \mathrm{HCO}_{3}(2.7 \mathrm{M})$ solution via sonication at ice bath conditions. The free $\left(\mathrm{NH}_{4}\right)_{2} \mathrm{SO}_{4}$ or $\mathrm{NH}_{4} \mathrm{HCO}_{3}$ was removed by dialysis against $10 \mathrm{wt} \%$ sucrose solution with $5 \mathrm{mM} \mathrm{NaCl}$. Free Cypate was removed using dialysis and centrifugation. DOX was added to the liposome solution at a drug/lipid ratio of $0.1(\mathrm{w} / \mathrm{w})$ and incubated at room temperature for $24 \mathrm{~h}$. Free DOX was removed using dialysis in distilled water for $9 \mathrm{~h}$. $\left(\mathrm{NH}_{4}\right)_{2} \mathrm{SO}_{4}$ liposomes were abridged for $\mathrm{L}$. The composition of the liposomes is listed in Table 1.

Table I Composition of DOX-L, DOX-BTSL, Cypate/DOX-L, and Cypate/DOX-BTSL

\begin{tabular}{lll}
\hline Liposomes & $\begin{array}{l}\text { DPPC:Chol:DSPE- } \\
\text { PEG }_{2000} \text { :Cypate } \\
\text { (molar ratio) }\end{array}$ & $\begin{array}{l}\text { DOX:lipid } \\
\text { (mass ratio) }\end{array}$ \\
\hline DOX-L & $12: 8: 1: 0$ & $1: 10$ \\
DOX-BTSL & $12: 8: 1: 0$ & $1: 10$ \\
Cypate/DOX-L & $12: 8: 1: 0.8$ & $1: 10$ \\
Cypate/DOX-BTSL & $12: 8: 1: 0.8$ & $1: 10$ \\
\hline
\end{tabular}

Abbreviations: DOX, doxorubicin; $\mathrm{L},\left(\mathrm{NH}_{4}\right)_{2} \mathrm{SO}_{4}$ liposomes; BTSL, bubblegenerating thermosensitive liposomes $\left(\mathrm{NH}_{4} \mathrm{HCO}_{3}\right)$; DPPC, dipalmitoylphosphatidylcholine; Chol, cholesterol; DSPE-PEG 2000 , I,2-distearyl-sn-glycero-3-phosphoethanaolamine- $\mathrm{N}$-[methoxypoly-(ethylene glycol)-2000].

\section{Characterization of liposomes}

The mean diameter, polydispersity index (PDI), and zeta potential were measured by dynamic light scattering (DLS; Zetasizer Nano-ZS, Malvern Instruments Ltd., Worcestershire, UK). The encapsulated DOX concentration was determined by fluorescence spectrophotometer (F-7000; Hitachi, Tokyo, Japan) after destroying the liposomes with $10 \%$ Triton X-100. The encapsulation efficiency (EE) and drug loading (DL) content of DOX were calculated according to the following equation:

$$
\begin{gathered}
\text { EE }(\%)=\frac{\text { Mass of DOX in liposomes }}{\text { Mass of DOX in the initial solution }} \times 100 \\
\text { DL }(\%)=\frac{\text { Mass of DOX in liposomes }}{\text { Mass of liposomes }} \times 100
\end{gathered}
$$

\section{Aqueous stability and photostability}

The fluorescent and ultraviolet (UV) stability of Cypate/ DOX-BTSL and free Cypate $(2.5 \mu \mathrm{g} / \mathrm{mL})$ were evaluated in phosphate-buffered saline (PBS) solution ( $\mathrm{pH} 7.4$ and 5.0). Size stability of $200 \mu \mathrm{g} / \mathrm{mL}$ samples was also measured by DLS at different timings in PBS solution ( $\mathrm{pH}$ 7.4). To assess the photostability, Cypate/DOX-BTSL, Cypate/ DOX-L, and free Cypate $(2.5 \mu \mathrm{g} / \mathrm{mL})$ were irradiated at $2.8 \mathrm{~W} / \mathrm{cm}^{2}$ (785 nm, MW-IR-785/1-2,500 mW; Changchun Femtosecond Technology Co., Ltd., Changchun, People's Republic of China) for 1, 2, 3, 5, 7, and $10 \mathrm{~min}$. Then, the UV absorbance of Cypate was measured at $780 \mathrm{~nm}$.

\section{Photothermal effect}

The solution of Cypate/DOX-BTSL and free Cypate (each $2.5 \mathrm{~mL}$ ) with various concentrations of Cypate was irradiated at the excitation wavelength of $785 \mathrm{~nm}\left(2.8 \mathrm{~W} / \mathrm{cm}^{2}\right)$ for $5 \mathrm{~min}$. The temperature of the solution was recorded for each $30 \mathrm{~s}$. PBS was used as the control in this experiment.

\section{In vitro release of DOX from liposomes}

The drug release behavior of DOX from the liposomes was observed by immersing the test liposomes $(10 \mathrm{mg} / \mathrm{mL}$, $2 \mathrm{~mL}$ ) in tube containing $10 \mathrm{~mL}$ PBS (pH 7.4 or 5.0), which were performed at $37^{\circ} \mathrm{C}$ or $42^{\circ} \mathrm{C}$ in an air-bath shaker at $100 \mathrm{rpm} .{ }^{27}$ In addition, DOX release behavior from liposomes with or without NIR irradiation $\left(785 \mathrm{~nm}, 2.8 \mathrm{~W} / \mathrm{cm}^{2}\right)$ for $10 \mathrm{~min}$ at initial time was also investigated to assess the photothermal-induced drug release. Samples of $2.0 \mathrm{~mL}$ were taken and replaced with an equal volume of fresh PBS at the definite time intervals. The amount of DOX released was determined using a fluorescence spectrometer $(E x=485 \mathrm{~nm}$, $\mathrm{Em}=580 \mathrm{~nm}$ ) at each time intervals. 


\section{Cellular uptake}

MCF-7 cells were seeded at a density of $2 \times 10^{5}$ cells/well in the confocal dishes and incubated overnight in RPMI 1640 containing $10 \%$ fetal bovine serum. Then, the medium was replaced with free DOX, Cypate/DOX-L, and Cypate/ DOX-BTSL dissolved in RPMI 1640 for $2 \mathrm{~h}$. After incubation, the medium was irradiated with NIR laser for $5 \mathrm{~min}$ and incubated for another $2 \mathrm{~h}$. The medium was removed, and the treated cells were washed with fresh PBS for three times to remove the test liposomes and free drugs that were not taken up by the cells. Then the cells were stained with 4', 6-diamidino-2-phenylindole (DAPI) for $20 \mathrm{~min}$ at room temperature and examined by a confocal laser scanning microscope (CLSM, FV1200; Olympus, Tokyo, Japan; Ex [485 nm] for DOX and Ex [633 nm] for Cypate).

\section{Acridine orange $(\mathrm{AO})$ staining}

$\mathrm{AO}$ fluorescent dyes were further used to assess the disruption of lysosomal membranes in MCF-7 cells. ${ }^{15}$ MCF-7 cells were seeded at a density of $2 \times 10^{5}$ cells/well at a 12 -well plate and then incubated overnight at $37^{\circ} \mathrm{C}$ with $5 \% \mathrm{CO}_{2}$. After treated with Cypate/DOX-L and Cypate/DOX-BTSL at the Cypate concentrations of $0.5,1$, and $2 \mu \mathrm{g} / \mathrm{mL}$ for $4 \mathrm{~h}$, the cells were exposed to photoirradiation for $10 \mathrm{~min}$ at $780 \mathrm{~nm}, 2.8 \mathrm{~W} / \mathrm{cm}^{2}$. The cells treated with PBS were as a control. After $2 \mathrm{~h}$, the cells were washed with PBS and stained with $200 \mu \mathrm{L}$ AO $(100 \mu \mathrm{g} / \mathrm{mL})$ for $15 \mathrm{~min}$ in dark and then immediately washed three times with PBS. Finally, the cells were observed using fluorescence microscopy (IX71-F22PH; Olympus) with an excitation wavelength of $488 \mathrm{~nm}$.

\section{MTT assay}

Cytotoxicity of the liposomes with or without NIR laser irradiation was assessed by a MTT method. MCF-7 cells were seeded into a 96-well plate at a density of $1 \times 10^{5}$ cells/well and then incubated overnight at $37^{\circ} \mathrm{C}$ with $5 \% \mathrm{CO}_{2}$. The cells were incubated with free DOX, Cypate/DOX-L, or Cypate/ DOX-BTSL for $4 \mathrm{~h}$ at various DOX concentrations of 20, 40, 60,80 , and $100 \mu \mathrm{g} / \mathrm{mL}$, followed by $10 \mathrm{~min}$ NIR irradiation $\left(785 \mathrm{~nm}, 2.8 \mathrm{~W} / \mathrm{cm}^{2}\right)$. The formulation without NIR irradiation was used as a control. After the further incubation for $2 \mathrm{~h}$, the cells were stained with $20 \mu \mathrm{L}$ MTT solution $(5.0 \mathrm{mg} / \mathrm{mL})$ for $4 \mathrm{~h}$, and the formed formazan crystals was dissolved with $150 \mu \mathrm{L}$ DMSO. Absorbance was recorded at $570 \mathrm{~nm}$ using a microplate reader (Infinite M200 Pro; Tecan, Männedorf, Switzerland).

\section{Trypan blue staining}

The effect of Cypate/DOX-L and Cypate/DOX-BTSL on cell injury was also evaluated using trypan blue exclusion method. ${ }^{15}$ MCF-7 cells at a density of $1 \times 10^{5}$ cells/well were seeded into a 24-well plate and incubated overnight. After treated with PBS, Cypate/DOX-L and Cypate/DOX-BTSL at the Cypate concentration of 2, 5, and $10 \mu \mathrm{g} / \mathrm{mL}$ for $4 \mathrm{~h}$, the cells were suffered from NIR irradiation for $10 \mathrm{~min}$ $\left(785 \mathrm{~nm}, 2.8 \mathrm{~W} / \mathrm{cm}^{2}\right)$. After another $2 \mathrm{~h}$, the cells were washed three times with PBS, and $200 \mu \mathrm{L}$ trypan blue solution $(0.1 \mathrm{wt} \%)$ was added. Then, the unstained living cells and stained dead cells were observed by an optical microscope (XDS-1B; Chongqing Optical \& Electrical Instrument Co., Ltd, Chongqing, People's Republic of China).

\section{Animals and tumor model}

Female BALB/c nude mice (4-6 wk old) were purchased from Beijing HFK Bioscience Co., Ltd. (Beijing, People's Republic of China). The protocols for the animal studies were approved by the Institutional Animal Ethical Committee of Fuzhou University, and the animal-handling procedures were according to the guidelines for the Care and Use of Laboratory Animals. Approximately $1 \times 10^{7} 4 \mathrm{~T} 1$ cells/each mouse were subcutaneously injected into the right side alar of the mice for constructing the tumor-bearing mice. ${ }^{28,29}$

\section{In vivo DOX accumulation}

The nude mice were chosen for the experiment when the tumor volume reached $80-100 \mathrm{~mm}^{3}$. The mice were randomly divided into three groups ( $n=4$ per group), and then the mice were treated with free DOX, Cypate/DOX-L, and Cypate/ DOX-BTSL (all containing $10 \mathrm{mg} / \mathrm{kg}$ DOX) via intratumoral injection, respectively. Tumor sites were irradiated with NIR for $5 \min \left(785 \mathrm{~nm}, 1.6 \mathrm{~W} / \mathrm{cm}^{2}\right)$ after administration of Cypate/DOX-L and Cypate/DOX-BTSL for $2 \mathrm{~h}$. Then, the mice were imaged using a CRI Maestro system (Maestro $^{\mathrm{TM}}$ 2 Maestro $\left.^{\mathrm{TM}} \mathrm{EX}\right)$ at $1,2,3$, and $4 \mathrm{~d}$ postinjection. The average fluorescent intensity at tumor was calculated at different timings. Moreover, the mice were sacrificed at $24 \mathrm{~h}$ after injection, and various organs including heart, liver, spleen, lung, kidney, and tumor were collected and imaged to monitor the in vivo DOX accumulation. ${ }^{30}$

\section{Antitumor efficacy study}

To evaluate the antitumor efficacy for each treatment, 24 tumor-bearing mice were randomly divided into six groups. The mice were treated with no treatment, free DOX, Cypate/ DOX-L (with and without laser), and Cypate/DOX-BTSL (with and without laser) at $10 \mathrm{mg} / \mathrm{kg}$ DOX via intratumoral injection. For the laser treatment groups, the tumor areas were exposed to photoirradiation for $5 \mathrm{~min}$ at the wavelength of $785 \mathrm{~nm}\left(1.6 \mathrm{~W} / \mathrm{cm}^{2}\right)$ at $1 \mathrm{~d}$. The tumor volume and 
body weight of the mice were measured every other day. The mice were killed after the experiments. Tumor volume was computed as (tumor length) $\times(\text { tumor width })^{2} / 2$.

\section{Statistical analysis}

All experiment data were expressed as mean \pm standard deviation (SD) and analyzed statistically by an one-way analysis of variance. The level of significance was defined at $P<0.05$.

\section{Results and discussion Preparation and characterization of liposomes}

To trigger a rapid release of drugs in tumor cells, a NIR responsive liposomal system was fabricated by thin-lipid film hydration and sonication. The key components of this new system were Cypate and $\mathrm{NH}_{4} \mathrm{HCO}_{3}$. Cypate exhibits NIRF and PTT after excitation by near-infrared light, which can trigger hyperthermia. ${ }^{31}$ Upon heating to a high temperature $\left(40^{\circ} \mathrm{C}-42^{\circ} \mathrm{C}\right), \mathrm{NH}_{4} \mathrm{HCO}_{3}$ encapsulated in the liposomes could quickly decompose to generate $\mathrm{CO}_{2}$ bubbles, leading to permeable defects in the lipid bilayers of the liposomes, facilitating the rapid local release of drugs (Figure 1).

Based on the transmembrane gradient of $\left(\mathrm{NH}_{4}\right)_{2} \mathrm{SO}_{4}$ or $\mathrm{NH}_{4} \mathrm{HCO}_{3}, \mathrm{DOX}$ was loaded into the test liposomes. Table 2 lists the characteristics of DOX-L, DOX-BTSL, Cypate/ DOX-L, and Cypate/DOX-BTSL. The mean diameters of DOX-L and DOX-BTSL were 133.6 \pm 2.8 and $126.7 \pm 5.2 \mathrm{~nm}$, and that of Cypate/DOX-L and Cypate/DOX-BTSL were $156.6 \pm 2.0$ and $156 \pm 3.2 \mathrm{~nm}$. The PDIs of the liposomes were all $<0.200$. These data showed a good homogeneity of the liposomes. The liposomes with these sizes could be internalized by tumor cells and accumulated in the deeper areas of tumor sites for cancer therapy by the EPR effect. ${ }^{32,33}$ The zeta potentials of liposomes were obviously negative in distilled water (Table 2), which suggested that these liposomal systems would be stable in aqueous media. ${ }^{34}$ Besides, as the crucial element for clinical application, an efficient loading of DOX into the aqueous phase of each formulation $(>84.5 \%)$ was achieved, and the DL contents were all $>6.93 \%$.

\section{Aqueous stability, photostability, and photothermal effect}

Cypate is a dicarboxylic acid-containing carbocyanine fluorophore with an excellent molar extinction coefficient and high photothermal conversion efficiency. The aqueous stability of Cypate was a prerequisite for cancer therapy; thus, we evaluated the aqueous stability of Cypate/DOX-BTSL and free Cypate solution. Figure 2A and B shows that the liposomes exhibit a good stability of Cypate in the fluorescence and UV absorbance at $\mathrm{pH} 5.0$ and $\mathrm{pH}$ 7.4, compared to free Cypate. It indicated that the liposomes could reduce the photobleaching of Cypate and maintain a stable structure in aqueous solution at acidic and physiological $\mathrm{pH}$, leading to the prevention of Cypate from the degradation in acidic endocytic compartments at tumor due to its efficient encapsulation in the liposomes. ${ }^{35}$ As shown in Figure 2C, the average diameters of Cypate/DOX-BTSL and Cypate/DOX-L remain around the initial sizes of liposomes without aggregation and precipitation in $10 \mathrm{~d}$, suggesting a great stability of Cypate/ DOX-BTSL and Cypate/DOX-L.

The photostability of liposomes and free Cypate was also demonstrated since it significantly affected the efficiency of PTT. According to the UV spectra of Cypate/DOX-BTSL, Cypate/DOX-L, and free Cypate (Figure 2D), the absorbance of free Cypate decreased quickly under $785 \mathrm{~nm}$ photoirradiation within 3 min due to the rapid photobleaching. Nevertheless, Cypate in the liposomes had much slower decrease of the absorbance under the same condition during $10 \mathrm{~min}$. It implied that the liposomes could improve the photostability of Cypate and provide more efficient PTT during longer irradiation time as compared to free Cypate.

To assess the photothermal effect of Cypate/DOX-BTSL and free Cypate, their thermal behaviors at different concentrations of Cypate in PBS (pH 7.4) under NIR irradiation were

Table 2 Characteristics of DOX-L, DOX-BTSL, Cypate/DOX-L, and Cypate/DOX-BTSL

\begin{tabular}{|c|c|c|c|c|c|}
\hline Liposomes & $\begin{array}{l}\text { Diameter } \\
(\mathrm{nm})^{\mathrm{a}}\end{array}$ & $\mathbf{P D I}^{\mathbf{a}}$ & $\begin{array}{l}\text { Zeta potential } \\
(\mathrm{mV})^{\mathrm{a}}\end{array}$ & EE (\%) ${ }^{b}$ & DL (\%) \\
\hline DOX-L & $133.6 \pm 2.8$ & $0.200 \pm 0.003$ & $-21.6 \pm 0.38$ & $84.8 \pm 2.39$ & $6.93 \pm 0.89$ \\
\hline DOX-BTSL & $126.7 \pm 5.2$ & $0.095 \pm 0.008$ & $-24.9 \pm 0.16$ & $91.14 \pm 3.0$ & $10.3 \pm 0.38$ \\
\hline Cypate/DOX-L & $156.6 \pm 2.0$ & $0.098 \pm 0.007$ & $-32.8 \pm 0.20$ & $84.5 \pm 2.60$ & $14.47 \pm 1.26$ \\
\hline Cypate/DOX-BTSL & $156 \pm 3.2$ & $0.070 \pm 0.005$ & $-26.8 \pm 0.09$ & $96.8 \pm 5.6$ & $10.7 \pm 0.57$ \\
\hline
\end{tabular}

Notes: ${ }^{a}$ The diameter, PDI, and zeta potential (mean $\pm \mathrm{SD}$ ) measured by DLS three times. ${ }^{\mathrm{b}} \mathrm{EE}$ and $\mathrm{DL}$ content of DOX (mean \pm SD) determined by fluorescence spectrometer three times.

Abbreviations: DOX, doxorubicin; $\mathrm{L},\left(\mathrm{NH}_{4}\right)_{2} \mathrm{SO}_{4}$ liposomes; $\mathrm{BTSL}$, bubble-generating thermosensitive liposomes $\left(\mathrm{NH}_{4} \mathrm{HCO}\right)$; $\mathrm{PDI}$, polydispersity index; $\mathrm{EE}$, entrapment efficiency; DL, drug loading; SD, standard deviation. 

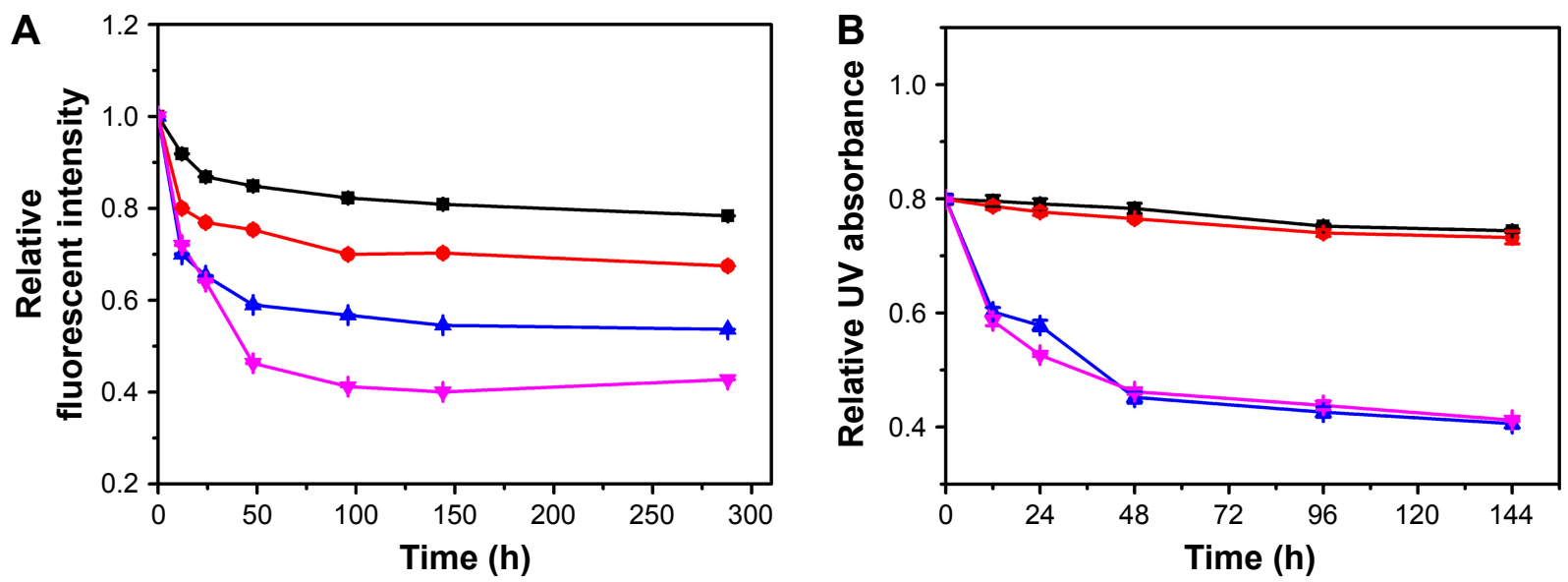

Cypate/DOX-BTSL at pH $7.4 \multimap-$ Cypate/DOX-BTSL at pH $5.0 \multimap$ Free Cypate at pH $7.4 \rightarrow$ Free Cypate at pH 5.0
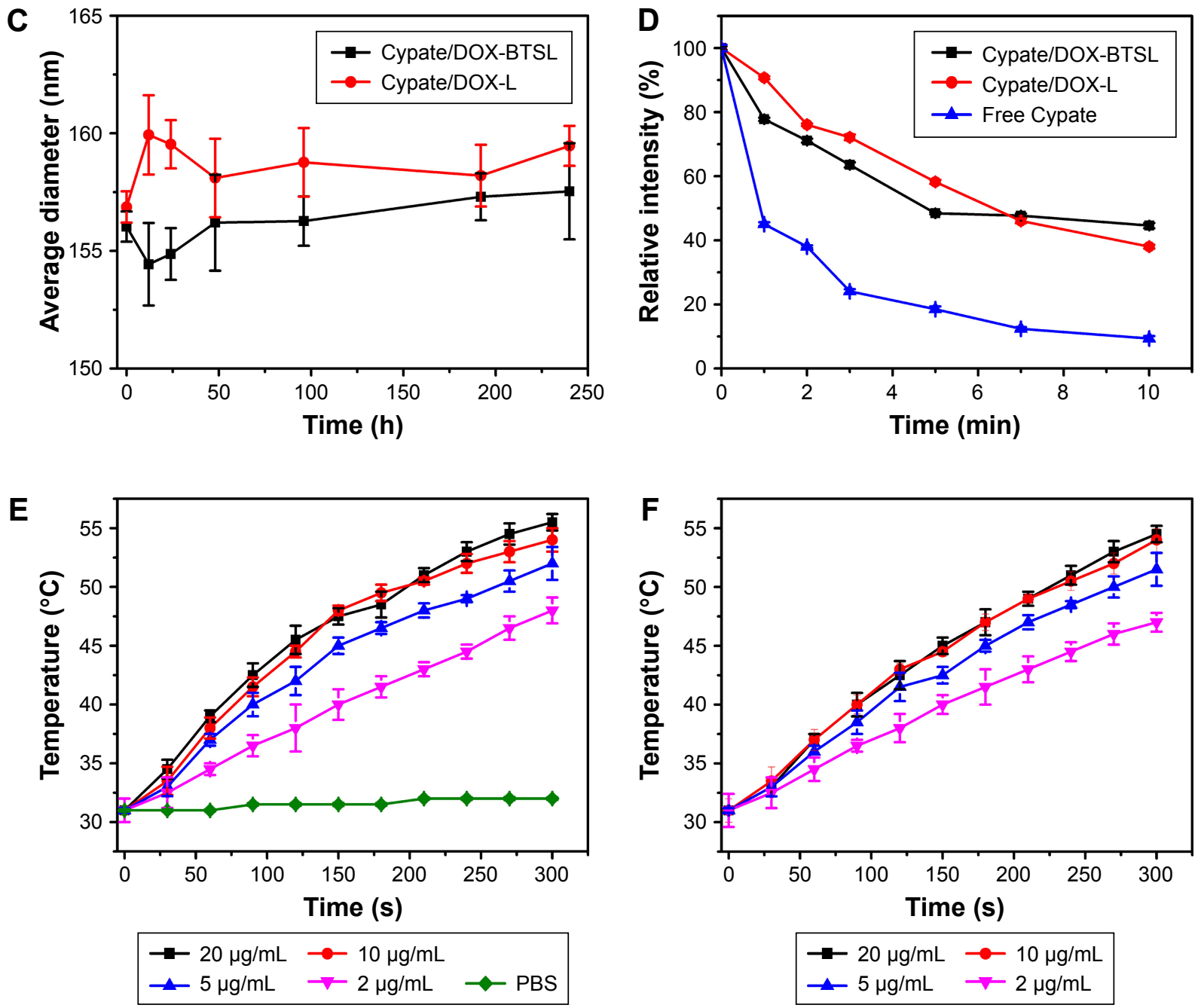

Figure 2 Relative fluorescent intensity (A) and UV absorbance (B) of Cypate/DOX-BTSL and free Cypate solution at pH 5.0 and 7.4. (C) Size change of Cypate/DOXBTSL and Cypate/DOX-L solution. (D) UV absorbances of Cypate/DOX-BTSL, Cypate/DOX-L, and free Cypate at different timings under $785 \mathrm{~nm}$ photoirradiation. Photothermal effect of free Cypate (E) and Cypate/DOX-BTSL (F) at various concentrations of Cypate within 5 min photoirradiation. The data are shown as mean \pm SD $(n=3)$.

Abbreviations: UV, ultraviolet; DOX, doxorubicin; BTSL, bubble-generating thermosensitive liposomes $\left(\mathrm{NH}_{4} \mathrm{HCO}_{3}\right)$; $\mathrm{L},\left(\mathrm{NH}_{4}\right)_{2} \mathrm{SO}$ liposomes; PBS, phosphate-buffered saline; SD, standard deviation. 
measured. At the concentration of $2.0 \mu \mathrm{g} / \mathrm{mL}$ Cypate, free Cypate, and Cypate/DOX-BTSL all displayed an obvious increase in the temperature from $31^{\circ} \mathrm{C}$ to $42^{\circ} \mathrm{C}$ with the NIR irradiation $\left(2.8 \mathrm{~W} / \mathrm{cm}^{2}\right)$ for $180 \mathrm{~s}$, whereas PBS as a control did not show any increase in temperature (Figure 2E and F). It suggested that Cypate/DOX-BTSL could quickly trigger hyperthermia at a low concentration of Cypate within a short photoirradiation, which was similar to the result of free Cypate. Moreover, it showed that the higher concentration of Cypate could cause more significant hyperthermia. These results indicated that Cypate/DOX-BTSL exhibited a concentrationdependent photothermal effect and had a potential to trigger photothermal damage against tumor cells $\left(>42^{\circ} \mathrm{C}\right) .{ }^{36,37}$

\section{In vitro release of DOX from liposomes}

To access the effect of $\mathrm{NH}_{4} \mathrm{HCO}_{3}$ on the rapid release of drugs from liposomes, the release profiles of DOX from DOXBTSL and DOX-L were evaluated at $37^{\circ} \mathrm{C}$ (physiological temperature) and $42^{\circ} \mathrm{C}$ (hyperthermic temperature), respectively. As shown in Figure 3A, the amounts of DOX released from DOX-L and DOX-BTSL are 20\%-26\% in PBS (pH 5.0 or 7.4 ) at $37^{\circ} \mathrm{C}$ during $24 \mathrm{~h}$, suggesting that the release rate of DOX was rather slow and the liposomes were all relatively stable at physiological temperature in $\mathrm{pH} 5.0$ and 7.4. The DOX release rate of DOX-L at $42^{\circ} \mathrm{C}$ (Figure 3B) was similar to that at $37^{\circ} \mathrm{C}$, which indicated that its drug release was not affected by temperature. However, the amounts of DOX released from DOX-BTSL were 84\% (pH 5.0) and 50\% (pH 7.4) within $30 \mathrm{~min}$ at $42^{\circ} \mathrm{C}$, which was obviously higher than that from DOX-L, implying that the release of DOX-BTSL had a temperature-dependent characteristic due to $\mathrm{NH}_{4} \mathrm{HCO}_{3}$. The reason of the rapid release was that the decomposition of $\mathrm{NH}_{4} \mathrm{HCO}_{3}$ at $42^{\circ} \mathrm{C}$ generated a large number of $\mathrm{CO}_{2}$ bubbles, which grew rapidly and then collapsed violently, ultimately resulting in a cavitation force that could perforate the lipidbilayer membranes and trigger a significant release of drugs. ${ }^{11}$ Furthermore, at $\mathrm{pH}$ 5.0, corresponding to the $\mathrm{pH}$ values of lysosome, DOX-BTSL displayed the faster drug release rate than that at $\mathrm{pH} 7.4$ at $42^{\circ} \mathrm{C}$, indicating that the release from DOX-BTSL exhibited a $\mathrm{pH}$-sensitive drug release property and facilitated the rapid release of DOX in the lysosome system at hyperthermic temperature.

In this study, the effect of laser irradiation on drug release behavior from Cypate/DOX-BTSL was also investigated. After laser irradiation, the total DOX released from Cypate/ DOX-BTSL was $88 \%$ at $\mathrm{pH} 5.0$ and $56 \%$ at $\mathrm{pH} 7.4$ during 10 min (Figure 3C). It suggested that the NIR irradiation could trigger the rapid drug release from Cypate/DOX-BTSL and the release had a $\mathrm{pH}$-sensitive property. The reason of this photothermal-induced rapid release of drugs was that Cypate encapsulated in liposomes could trigger the hyperthermia under photoirradiation, inducing the decomposition of $\mathrm{NH}_{4} \mathrm{HCO}_{3}$ and the generation of $\mathrm{CO}_{2}$ bubbles.

Therefore, it could be concluded that Cypate/DOXBTSL had photothermal-induced and $\mathrm{pH}$-sensitive drug release properties. BTSL exhibited a good stability at $37^{\circ} \mathrm{C}$ during blood circulation. However, when the hyperthermia $\left(42^{\circ} \mathrm{C}\right)$ was reached by NIR irradiation or local heating, the drug release would be extremely fast, causing a high drug concentration gradient to facilitate the diffusion of drug into the tumor cells. The fast-triggered drug release also acquired the ability to counteract rapid passage of the bloodstream and prevent the liposomes from washout from the tumors. ${ }^{38}$

\section{In vitro cellular uptake}

The cellular uptake of free DOX, Cypate/DOX-L, and Cypate/DOX-BTSL was investigated by CLSM. It has been known that free DOX molecules are transported into the cells through a passive diffusion pathway, whereas liposomes are taken up by cells via an endocytotic pathway and then escape from the endosomes and/or the lysosomes to enter the cytoplasm. ${ }^{39}$ As shown in Figure 4, free DOX and DOX released from Cypate/DOX-L and Cypate/DOX-BTSL are all localized in the nucleus after $4 \mathrm{~h}$ incubation. This demonstrated that DOX has been released from liposomes and accumulated in the nucleus after $4 \mathrm{~h}$. Figure 4 exhibits that the group of Cypate/DOX-BTSL with NIR irradiation has the strongest red fluorescence in the nucleus, compared to those of Cypate/DOX-BTSL without NIR irradiation and Cypate/DOX-L, which was approximately to free DOX. However, red fluorescence distribution had no significant difference between Cypate/DOX-L group with and without irradiation. These data indicated that Cypate/DOX-BTSL with NIR irradiation improved the intracellular uptake of DOX clearly. It was due to the photothermal-induced rapid release of DOX from Cypate/DOX-BTSL activated by the formation of $\mathrm{CO}_{2}$ bubbles via the thermal decomposition of the encapsulated $\mathrm{NH}_{4} \mathrm{HCO}_{3}$, which was consistent with the results of in vitro release.

\section{Disruption of lysosomal membranes}

Photothermal damage on tumor cells is usually on account of the destabilization of subcellular organelles such as 

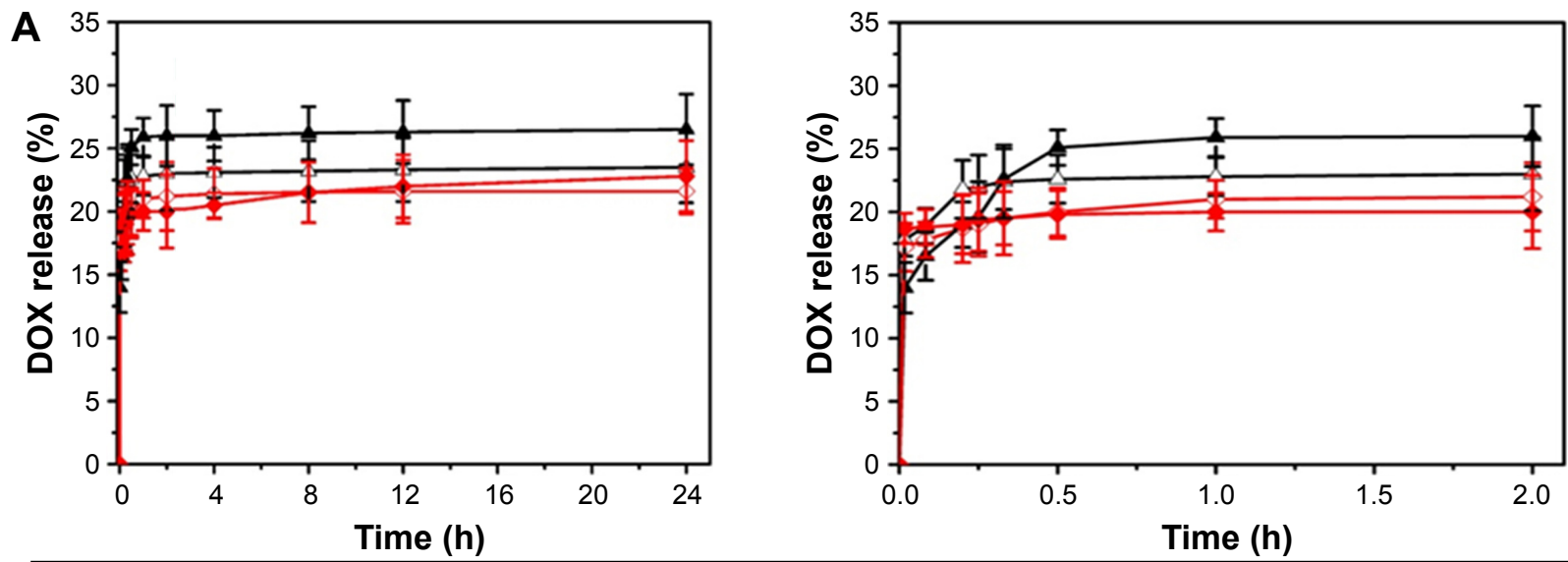

DOX-BTSL at $37^{\circ} \mathrm{C} \mathrm{pH}=5.0 \sim$ DOX-L at $37^{\circ} \mathrm{C} \mathrm{pH}=5.0 \multimap$ DOX-BTSL at $37^{\circ} \mathrm{C} \mathrm{pH}=7.4 \prec$ DOX-L at $37^{\circ} \mathrm{C} \mathrm{pH}=7.4$
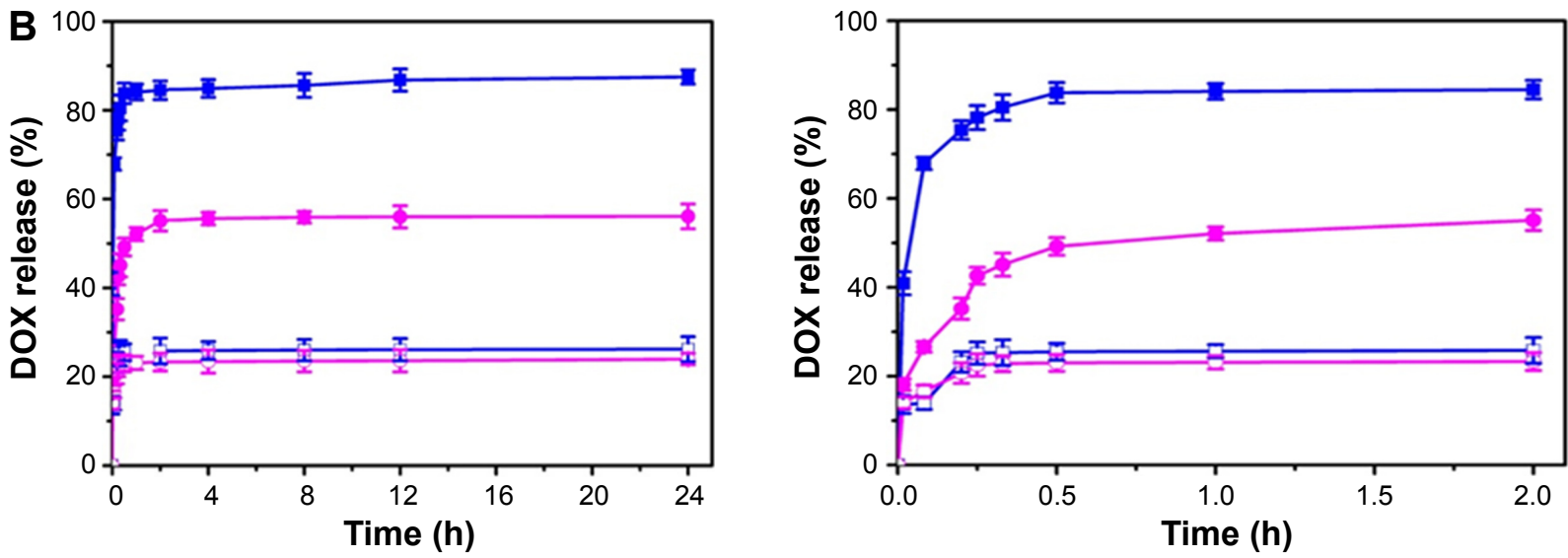

- DOX-BTSL at $42^{\circ} \mathrm{C} \mathrm{pH}=5.0 \multimap-\mathrm{DOX}-\mathrm{L}$ at $42^{\circ} \mathrm{C} \mathrm{pH}=5.0 \multimap-\mathrm{DOX}-\mathrm{BTSL}$ at $42^{\circ} \mathrm{C} \mathrm{pH}=7.4 \multimap-\mathrm{DOX}-\mathrm{L}$ at $42^{\circ} \mathrm{C} \mathrm{pH}=7.4$

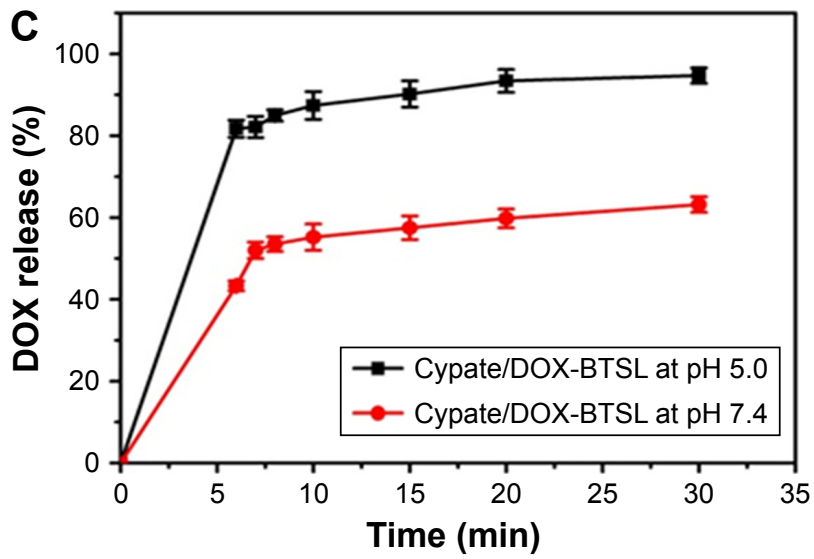

Figure 3 Release profiles of DOX from the DOX-BTSL and DOX-L incubated in PBS (pH 5.0 and 7.4$)$ at $37^{\circ} \mathrm{C}(\mathbf{A})$ and $42^{\circ} \mathrm{C}(\mathbf{B})$, respectively. The right-hand side of (A) and (B) displays a zoom-in image of drug release in the first $2 \mathrm{~h}$. (C) DOX release profiles from Cypate/DOX-BTSL in PBS (pH 5.0 and 7.4) after laser irradiation. The data are shown as mean $\pm S D(n=3)$.

Abbreviations: DOX, doxorubicin; BTSL, bubble-generating thermosensitive liposomes $\left(\mathrm{NH}_{4} \mathrm{HCO}_{3}\right)$; $\mathrm{L}$, $\left(\mathrm{NH}_{4}\right)_{2} \mathrm{SO}_{4}$ liposomes; $\mathrm{PBS}$, phosphate-buffered saline; $\mathrm{SD}$, standard deviation.

lysosomes. To investigate the disruption of lysosomal membranes, we monitored the integrity of lysosomes with photoirradiation using $\mathrm{AO}$ as an intracellular indicator. $\mathrm{AO}$ is a metachromatic fluorophore that becomes charged and is retained by proton trapping within acidic compartments.
$\mathrm{AO}$ emits an intense red fluorescence in intact acidic lysosomes and displays a green fluorescence in neutralized nuclei and cytosol. When acidic compartment such as lysosomes is disrupted, the red fluorescence disappears and only the green fluorescence can be observed. ${ }^{40}$ As shown in 


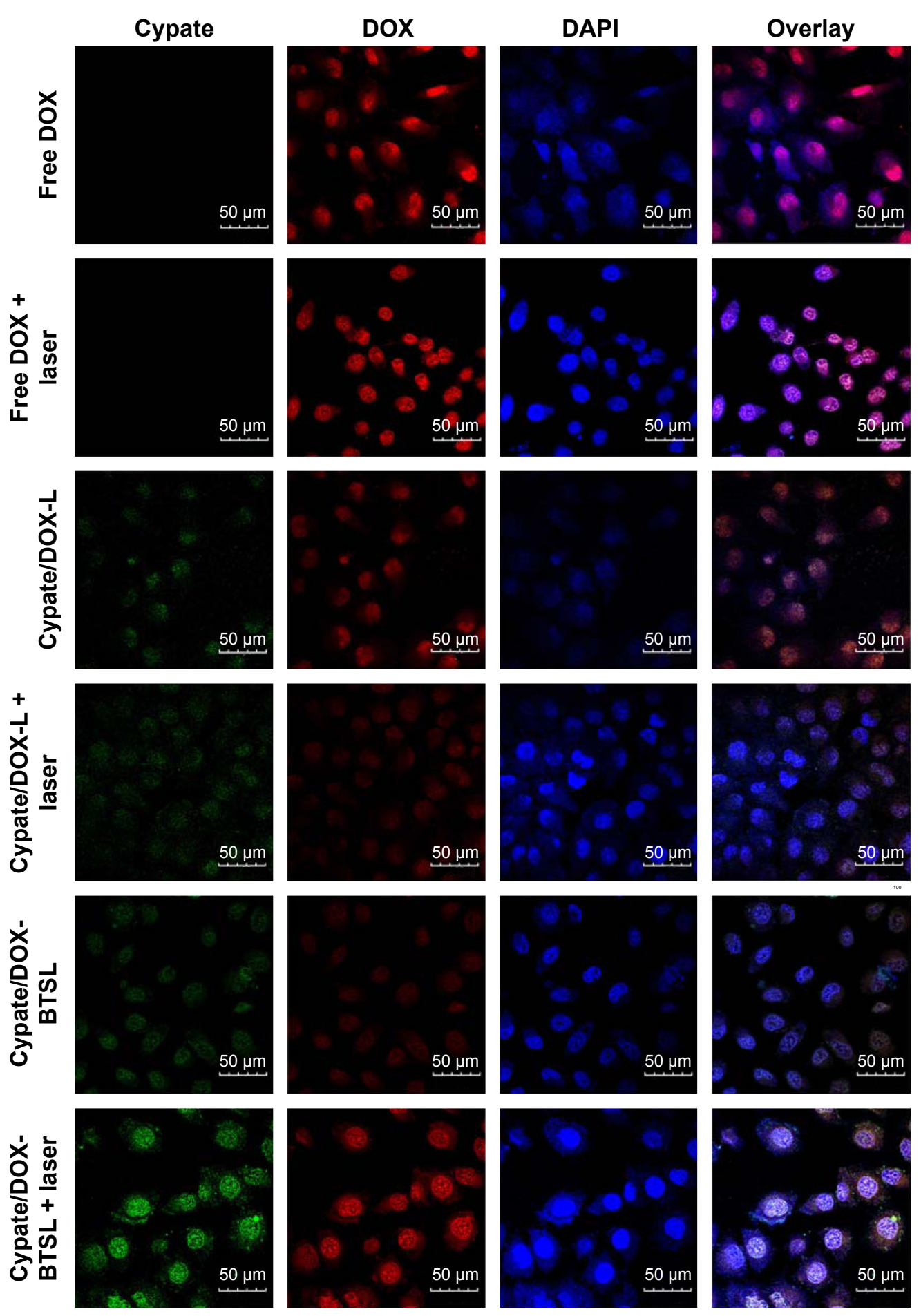

Figure 4 Confocal laser scanning microscopy of MCF-7 cells after $4 \mathrm{~h}$ incubation with free DOX, Cypate/DOX-L, and Cypate/DOX-BTSL with or without NIR laser irradiation.

Notes: Cypate, green fluorescence; DOX, red fluorescence; DAPI, blue fluorescence. Scale bars represent $50 \mu \mathrm{m}$ and magnification $\times 40$. Abbreviations: DOX, doxorubicin; $\mathrm{L},\left(\mathrm{NH}_{4}\right)_{2} \mathrm{SO}_{4}$ liposomes; $\mathrm{BTSL}$, bubble-generating thermosensitive liposomes $\left(\mathrm{NH}_{4} \mathrm{HCO}_{3}\right)$.

Figure 5, the acidic lysosomes in MCF-7 cells treated with PBS exhibit yellow fluorescence (overlay of red and green) with or without NIR irradiation, which indicated that PBS upon irradiation could not break the lysosomal membranes. Nevertheless, the red fluorescence from AO decreased in the presence of Cypate/DOX-L and Cypate/DOX-BTSL with irradiation, compared to the darkness group at the same dose of Cypate. The results implied that Cypate/DOX-L and Cypate/DOX-BTSL upon irradiation could disrupt the lysosomal membranes and realize endolysosomal escape into 


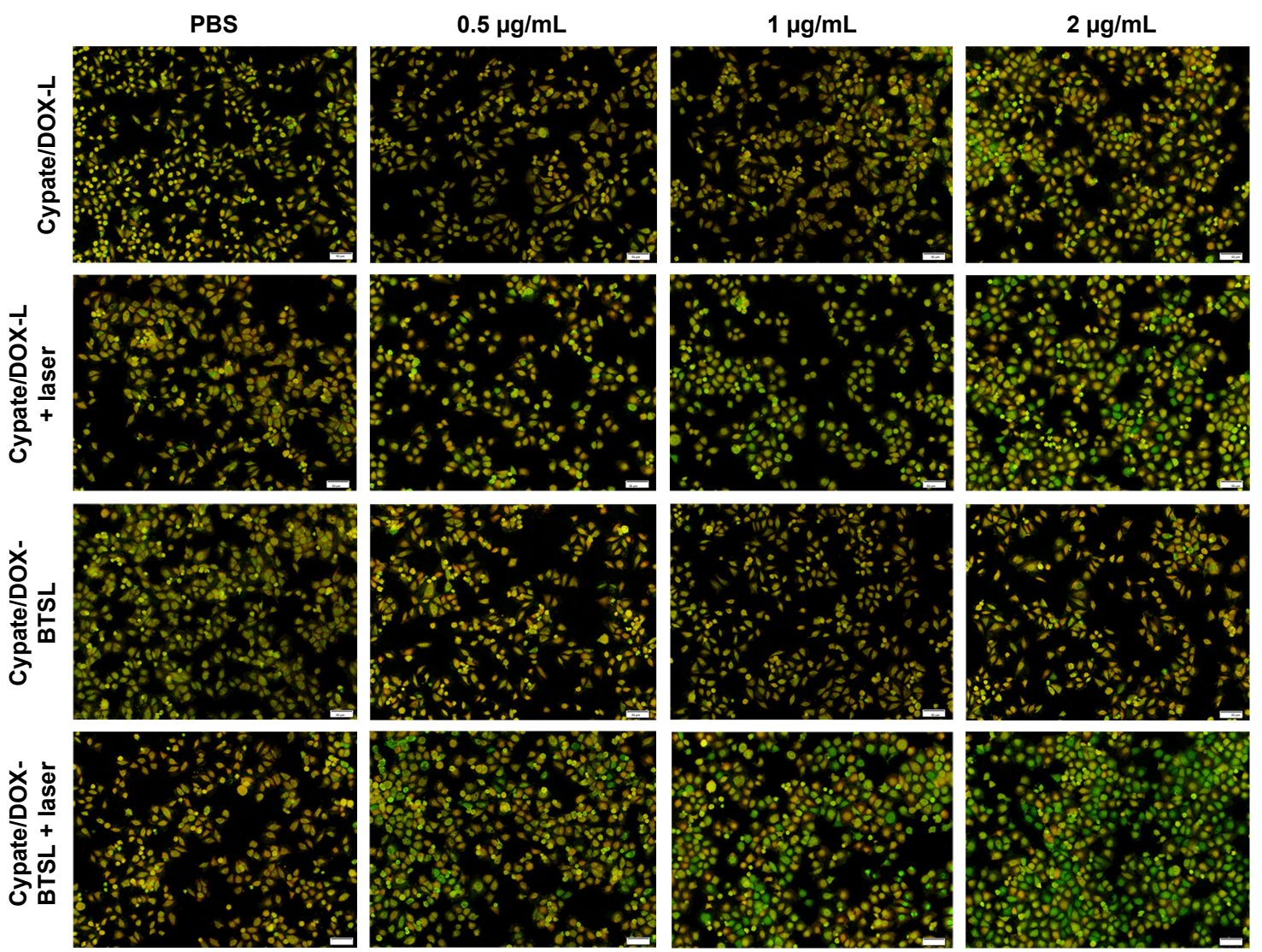

Figure 5 Observation of lysosomal disruption of MCF-7 cells treated with PBS, Cypate/DOX-L, and Cypate/DOX-BTSL at the Cypate concentrations of 0.5 , I, and $2 \mu g / \mathrm{mL}$ with or without laser irradiation using $A O$ staining. Scale bars represent $50 \mu \mathrm{m}$ and magnification $\times 12.6$.

Abbreviations: PBS, phosphate-buffered saline; DOX, doxorubicin; $\mathrm{L},\left(\mathrm{NH}_{4}\right)_{2} \mathrm{SO}_{4}$ liposomes; $\mathrm{BTSL}$, bubble-generating thermosensitive liposomes $\left(\mathrm{NH}_{4} \mathrm{HCO}{ }_{3}\right) ; \mathrm{AO}$, acridine orange.

cytosol, which was attributed to photothermal cytotoxicity. In addition, compared to Cypate/DOX-L with irradiation, the red fluorescence obviously decreased in Cypate/DOXBTSL laser group at the same concentration of Cypate. The red fluorescence significantly decreased in the presence of Cypate/DOX-BTSL $(0.5 \mu \mathrm{g} / \mathrm{mL}$ Cypate) with irradiation and mostly disappeared at the Cypate concentration of $2 \mu \mathrm{g} / \mathrm{mL}$. It suggested that Cypate/DOX-BTSL at a low concentration of Cypate could effectively damage the lysosomal membranes under irradiation. It indicated that the disruption of lysosomal membranes was owing to not only photothermal effect from Cypate but also the collapse of the $\mathrm{CO}_{2}$ bubbles. ${ }^{11}$ These could facilitate the DOX release to nucleus and then enhance the synergistic cytotoxicity of chemotherapy and PTT.

\section{Photothermal cytotoxicity}

The photothermal injury of Cypate/DOX-L and Cypate/ DOX-BTSL on MCF-7 cells was investigated by MTT. Figure 6 displays cell viability of MCF-7 cells treated

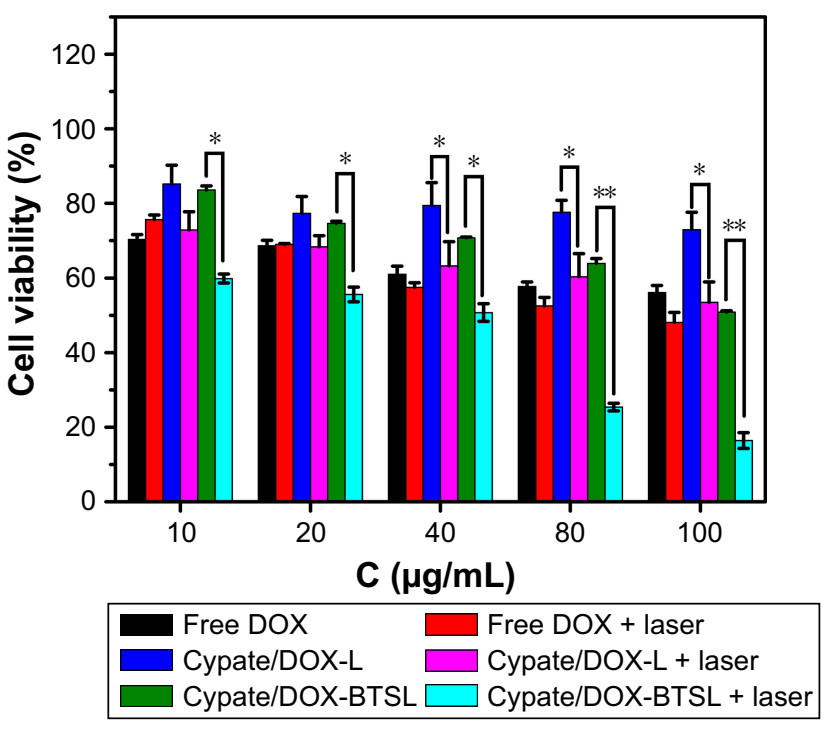

Figure 6 Cell viability of MCF-7 cells treated with free DOX, Cypate/DOX-L, and Cypate/DOX-BTSL at various concentrations of DOX with or without NIR laser irradiation.

Note: The error bars represent mean $\pm S D(n=5, * P<0.05$, $* * P<0.01)$.

Abbreviations: DOX, doxorubicin; $\mathrm{L},\left(\mathrm{NH}_{4}\right)_{2} \mathrm{SO}_{4}$ liposomes; BTSL, bubblegenerating thermosensitive liposomes $\left(\mathrm{NH}_{4} \mathrm{HCO}_{3}\right)$; $\mathrm{SD}$, standard deviation. 
with free DOX, Cypate/DOX-L, and Cypate/DOX-BTSL at various concentrations of DOX with or without NIR irradiation. With the increase in DOX concentration, the cell viability of all groups without irradiation was decreased since the cytotoxicity was improved due to chemotherapy. The cell viability of Cypate/DOX-L and Cypate/DOX-BTSL groups with irradiation was much lower than that of groups without irradiation. It was known that free Cypate did not induce cell death without photoirradiation, but it could kill the cells with irradiation owing to photothermal effect. Therefore, the explanation of the high cytotoxicity of the laser groups was that Cypate encapsulated in the liposomes could induce hyperthermia $\left(42^{\circ} \mathrm{C}\right)$, resulting in severe photothermal damage on tumor cells. Moreover, it was seen that the Cypate/ DOX-BTSL groups with irradiation had the lowest cell viability. It was since the hyperthermia triggered the rapid release of DOX from the bubble liposomes, increasing the intracellular concentration of DOX, causing stronger growth inhibition effect on the cells.

The photothermal injury of Cypate/DOX-L and Cypate/ DOX-BTSL on MCF-7 cells was further validated by trypan blue staining. Trypan blue dye molecules can easily penetrate plasma membranes of dead cells to stain the nuclei, whereas viable cells cannot be stained. From Figure 7, the cell damage of Cypate/DOX-L and Cypate/DOX-BTSL with NIR irradiation is more severe than the groups without irradiation, and the photothermal injury of the laser groups increased clearly with the increase in the concentrations of Cypate. Moreover, the photothermal injury of Cypate/DOX-BTSL under irradiation was more obvious than Cypate/DOX-L laser group. PBS as a control exhibited no damage on cells with or without laser irradiation. These data indicated that the cells suffered from severe photothermal injury from Cypate/DOX-BTSL because of the bubbles, which were consistent with the result of MTT.

Based on the results of MTT and trypan blue staining, synergistic cytotoxicity of Cypate/DOX-BTSL was due to a combination of chemotherapy and PTT. Notably, the hyperthermia from Cypate not only led to PTT but also induced the rapid release of DOX from the bubble liposomes, enhancing the cytotoxicity significantly.

\section{In vivo DOX accumulation}

The aforementioned experimental results in vitro indicated that Cypate/DOX-BTSL with NIR irradiation improved the intracellular uptake of DOX clearly. The accumulation of DOX in tumor and organs with or without NIR laser irradiation was studied following an intratumoral injection of free DOX, Cypate/DOX-L, and Cypate/DOX-BTSL. According to

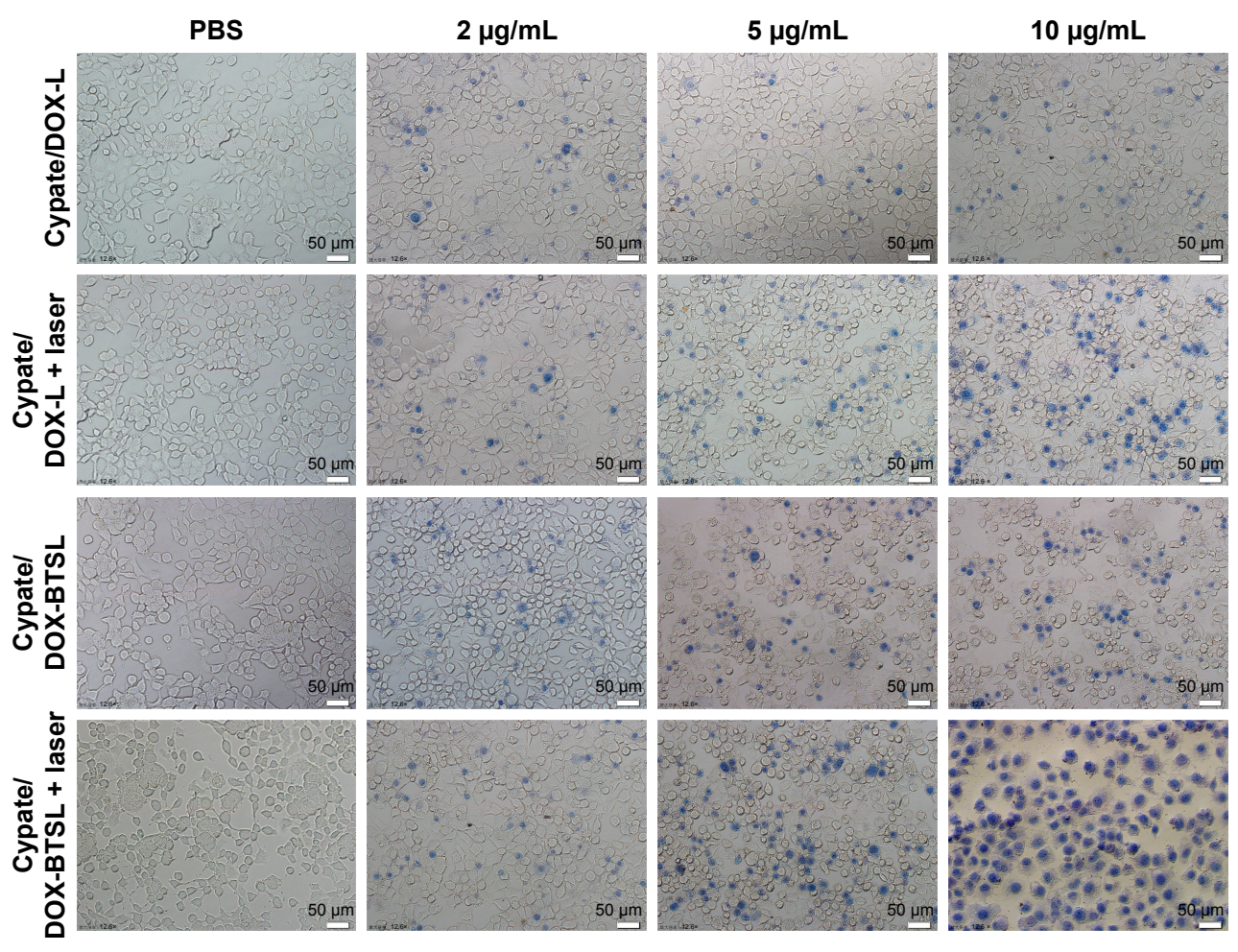

Figure 7 Trypan blue staining image of MCF-7 cells treated with PBS, Cypate/DOX-L, and Cypate/DOX-BTSL at the Cypate concentrations of 2, 5, and I0 $\mu$ g/mL with or without laser irradiation. Scale bars represent $50 \mu \mathrm{m}$ and magnification $\times 12.6$.

Abbreviations: PBS, phosphate-buffered saline; DOX, doxorubicin; $\mathrm{L},\left(\mathrm{NH}_{4}\right)_{2} \mathrm{SO}_{4}$ liposomes; $\mathrm{BTSL}$, bubble-generating thermosensitive liposomes $\left(\mathrm{NH}_{4} \mathrm{HCO}\right)$. 
Figure 8A, the fluorescent images of major organs and tumors ex vivo indicated that high drug concentration spread in lung and tumor at $24 \mathrm{~h}$ postinjection of free DOX, whereas Cypate/ DOX-L and Cypate/DOX-BTSL with laser irradiation presented high drug concentration only in tumor. It suggested that these liposomes could improve nonspecific accumulation of DOX and enrich the DOX in tumor owing to the EPR effect. The accumulations of DOX in tumor were further investigated at $1,2,3$, and $4 \mathrm{~d}$ postinjection by in vivo imaging (Figure $8 \mathrm{~B}$ and $\mathrm{C}$ ). In the laser treatment groups, the fluorescence intensities of DOX in tumor were stronger than that of free DOX group during the imaging period of $4 \mathrm{~d}$, and the DOX from
Cypate/DOX-L and Cypate/DOX-BTSL with irradiation was significantly retained around the tumor even after $4 \mathrm{~d}$, which was attributed to the prolonged retention time of DOX in tumor by EPR effects of liposomes. ${ }^{41}$ Compared to Cypate/DOX-L laser group, the Cypate/DOX-BTSL laser group exhibited stronger fluorescence intensity of DOX in tumor on the same condition. These results indicated that Cypate/DOX-BTSL dramatically increased the amount of DOX accumulated in tumor due to the photothermal-induced rapid release of DOX. Therefore, Cypate/DOX-BTSL would facilitate to prolong the retention of DOX in tumor, reduce systemic side effects, and enhance therapeutic efficacy of DOX.

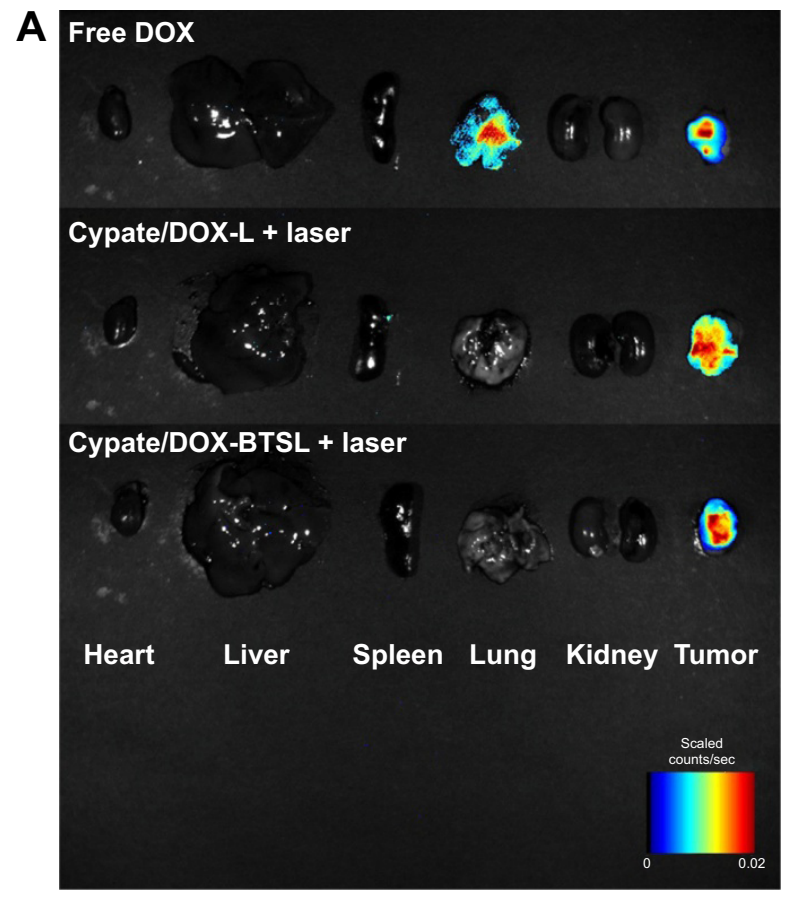

B
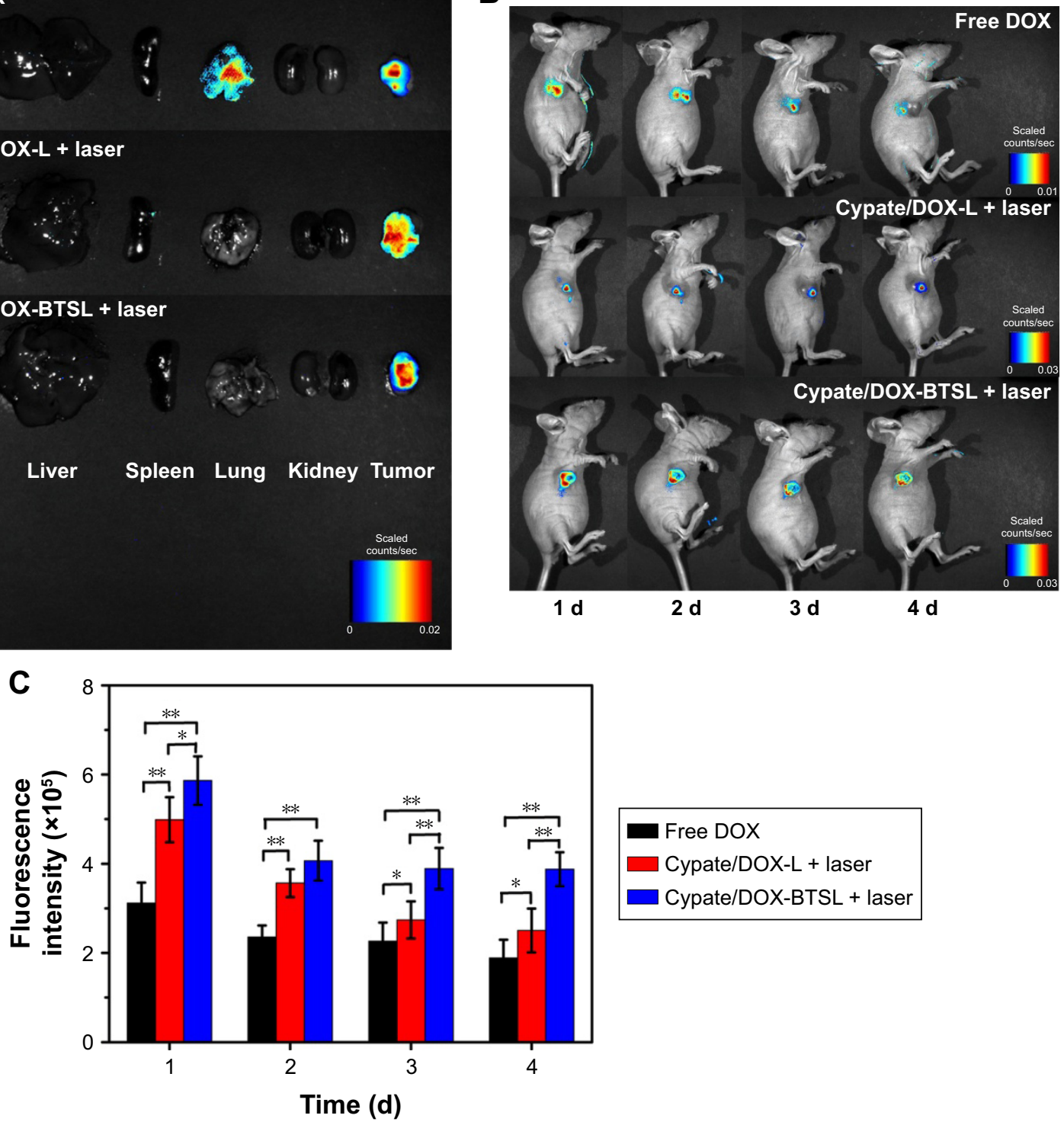

Figure 8 Ex vivo fluorescent images of major organs and tumors from the nude mice at 24 h postinjection $(\mathbf{A})$. In vivo imaging (B) and its fluorescent intensities (C) of the mice around the tumor at I, 2, 3, and $4 \mathrm{~d}$ postinjection, respectively. The data are shown as mean $\pm \mathrm{SD}(\mathrm{n}=4, * P<0.05, * * P<0.0 \mathrm{I})$.

Abbreviations: $\mathrm{SD}$, standard deviation; DOX, doxorubicin; $\mathrm{L},\left(\mathrm{NH}_{4}\right)_{2} \mathrm{SO}_{4}$ liposomes; $\mathrm{BTSL}$, bubble-generating thermosensitive liposomes $\left(\mathrm{NH}_{4} \mathrm{HCO}\right)_{3}$. 
A

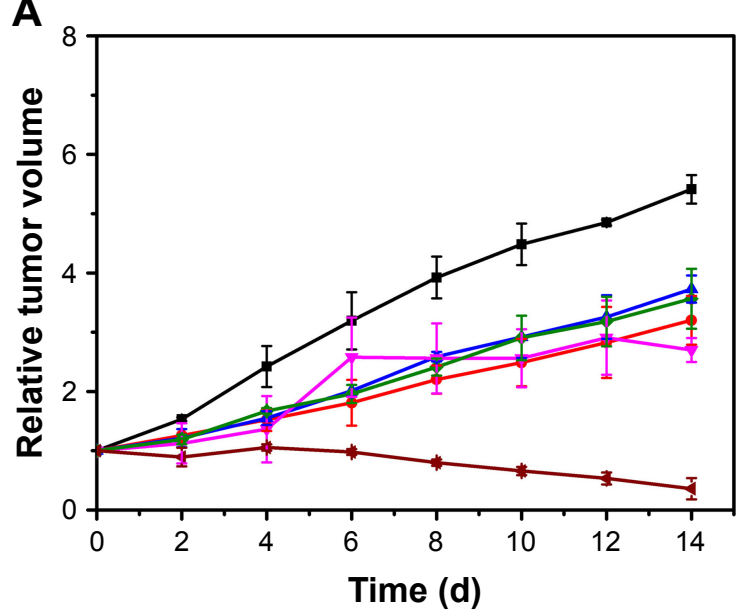

B

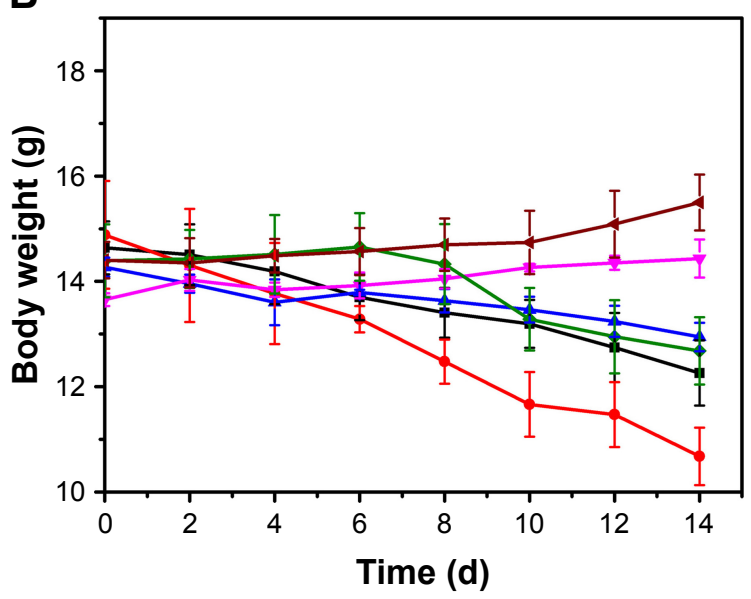

$\begin{array}{lll}\rightarrow \text { No treatment } & \rightarrow \text { Free DOX } & \leftarrow \text { Cypate/DOX-L } \\ \rightarrow \text { Cypate/DOX-L + laser } & \rightarrow \text { Cypate/DOX-BTSL } & \leftarrow \text { Cypate/DOX-BTSL + laser }\end{array}$

Figure 9 Changes in relative tumor volume $(\mathbf{A})$ and body weight $(\mathbf{B})$ of the nude mice-bearing $4 \mathrm{TI}$ tumors after treatment for $14 \mathrm{~d}$.

Note: The error bars represent mean \pm SD $(n=4)$.

Abbreviations: DOX, doxorubicin; $\mathrm{L},\left(\mathrm{NH}_{4}\right)_{2} \mathrm{SO}_{4}$ liposomes; BTSL, bubble-generating thermosensitive liposomes $\left(\mathrm{NH}_{4} \mathrm{HCO}\right)$; $\mathrm{SD}$, standard deviation.

\section{Antitumor activity}

The in vivo antitumor efficacy of Cypate/DOX-L and Cypate/DOX-BTSL in a synergistic therapy of PTT and chemotherapy was elucidated by the nude mice-bearing 4T1 tumors after treatment. From Figure 9A, the tumor volume of the mice with no treatment as control rapidly increases in $14 \mathrm{~d}$, while the tumor growth for free DOX and the liposome groups (with or without laser irradiation) is suppressed. For Cypate/DOX-L and Cypate/DOX-BTSL, the laser irradiation group had a better antitumor efficacy than the relative group without irradiation, implying that the effect of local photothermal heating could not be negligible. In the laser groups, Cypate/DOX-BTSL suppressed tumor growth more significantly than Cypate/DOX-L, since considerably more DOX molecules were released and accumulated in tumor due to the photothermal-induced rapid release of DOX from Cypate/DOX-BTSL. It was consistent with the previous results of in vitro release, cellular uptake, and in vivo imaging.

Furthermore, the body weight was measured to evaluate treatment-induced toxicity (Figure 9B). The mice treated with free DOX lost $>28 \%$ weight at $14 \mathrm{~d}$, indicating the serious toxic side effects of free DOX. ${ }^{32}$ The mice treated with the liposomes lost less origin weight and had a lower toxicity than the free DOX group. Notably, there was no significant reduction in body weight of the mice in the laser groups, implying the excellent safety of these liposomes under irradiation. These findings suggested that the synergistic therapy of PTT and chemotherapy of these liposomes could effectively inhibit tumor growth and reduce side effects of DOX, achieved a promising strategy for antitumor therapy.

\section{Conclusion}

A NIR responsive bubble-generating liposome (Cypate/ DOX-BTSL) was fabricated with $\mathrm{NH}_{4} \mathrm{HCO}_{3}$. Cypate/DOXBTSL exhibited a good aqueous stability, photostability, and photothermal effect. The liposomes had a property of photothermal-induced rapid drug release due to the hyperthermia induced by Cypate and $\mathrm{CO}_{2}$ bubbles triggered by $\mathrm{NH}_{4} \mathrm{HCO}_{3}$. After NIR irradiation, Cypate/DOX-BTSL could enhance the intracellular uptake and tumor accumulation of DOX significantly, compared to the groups without $\mathrm{NH}_{4} \mathrm{HCO}_{3}$. Therefore, this NIR responsive liposomal system shows a great perspective as a rapid drug release system for synergistic therapy of PTT and chemotherapy.

\section{Acknowledgments}

This research was supported by the National Natural Science Foundation of China (grant nos 81501576 and 51402294), the Natural Science Foundation of Fujian Province (grant no 2015J01341), the Science and Technology Development Foundation of Fuzhou University (grant no 2014-XY-14), and the Opening Project of Fujian Provincial Engineering Technology Research Center of Marine Functional Food (grant no C11178). 


\section{Disclosure}

The authors report no conflicts of interest in this work.

\section{References}

1. Sun TM, Du JZ, Yao YD, et al. Simultaneous delivery of siRNA and paclitaxel via a "two-in-one" micelleplex promotes synergistic tumor suppression. ACS Nano. 2011;5(2):1483-1494.

2. Mauceri HJ, Hanna NN, Beckett MA, et al. Combined effects of angiostatin and ionizing irradiation in antitumour therapy. Nature. 1998;394(6690):287-291.

3. Lane D. Designer combination therapy for cancer. Nat Biotechnol. 2006;24(2):163-164.

4. Yong KT, Roy I, Swihart M, Prasad P. Multifunctional nanoparticles as biocompatible targeted probes for human cancer diagnosis and therapy. J Mater Chem. 2009;19(27):4655-4672.

5. Westermann AM, Jones EL, Schem BC, et al. First results of triplemodality treatment combining radiotherapy, chemotherapy, and hyperthermia for the treatment of patients with stage IIB, III, and IVA cervical carcinoma. Cancer. 2005;104(4):763-770.

6. Huang XH, El-Sayed IH, Qian W, El-Sayed MA. Cancer cell imaging and photothermal therapy in the near-infrared region by using gold nanorods. J Am Chem Soc. 2006;128(6):2115.

7. Fang J, Nakamura H, Maeda H. The EPR effect: unique features of tumor blood vessels for drug delivery, factors involved, and limitations and augmentation of the effect. Adv Drug Deliv Rev. 2011;63(3):136-151.

8. Bozzuto G, Molinari A. Liposomes as nanomedical devices. Int $J$ Nanomed. 2015;10:975-999.

9. Shenoi MM, Shah NB, Griffin RJ, Vercellotti GM, Bischof JC. Nanoparticle pre-conditioning for enhanced thermal therapies in cancer. Cryobiology. 2011;63(3):315.

10. Zha ZB, Yue XL, Ren QS, Dai ZF. Uniform polypyrrole nanoparticles with high photothermal conversion efficiency for photothermal ablation of cancer cells. Adv Mater. 2013;25(5):777-782.

11. Carter KA, Shao S, Luo D, et al. Porphyrin-phospholipid liposomes permeabilized by near-infrared light. Nat Commun. 2014;5:3546.

12. Luo D, Carter KA, Razi A, et al. Doxorubicin encapsulated in stealth liposomes conferred with light-triggered drug release. Biomaterials. 2016;75:193-202.

13. Chung MF, Chen KJ, Liang HF, et al. A liposomal system capable of generating $\mathrm{CO}_{2}$ bubbles to induce transient cavitation, lysosomal rupturing, and cell necrosis. Angew Chem Int Ed. 2012;51(40):10089-10093.

14. Boddien A, Gartner F, Federsel C, et al. $\mathrm{CO}_{2}$-"neutral" hydrogen storage based on bicarbonates and formates. Angew Chem Int Ed. 2011;50(28):6411-6414.

15. Chen KJ, Liang HF, Chen HL, et al. A thermoresponsive bubblegenerating liposomal system for triggering localized extracellular drug delivery. ACS Nano. 2013;7(1):438-446.

16. Chen KJ, Chaung EY, Wey SP, et al. Hyperthermia-mediated local drug delivery by a bubble-generating liposomal system for tumor-specific chemotherapy. ACS Nano. 2014;8(5):5105-5115.

17. Yang H, Mao HJ, Wan ZH, et al. Micelles assembled with carbocyanine dyes for theranostic near-infrared fluorescent cancer imaging and photothermal therapy. Biomaterials. 2013;34(36):9124-9133.

18. Ye Y, Bloch S, Kao J, Achilefu S. Multivalent carbocyanine molecular probes: synthesis and applications. Bioconjug Chem. 2005;16(1):51-61.

19. Bahmani B, Lytle CY, Walker AM, Gupta S, Vullev VI, Anvari B. Effects of nanoencapsulation and PEGylation on biodistribution of indocyanine green in healthy mice: quantitative fluorescence imaging and analysis of organs. Int J Nanomed. 2013;8:1609-1620.

20. Almutairi A, Guillaudeu SJ, Berezin MY, Achilefu S, Frechet JM. Biodegradable $\mathrm{pH}$-sensing dendritic nanoprobes for near-infrared fluorescence lifetime and intensity imaging. $\mathrm{J} \mathrm{Am} \mathrm{Chem} \mathrm{Soc.} \mathrm{2008;}$ 130(2):444-445.

21. Peng CL, Shih YH, Lee PC, Hsieh TM, Luo TY, Shieh MJ. Multimodal image-guided photothermal therapy mediated by ${ }^{188}$ Re-labeled micelles containing a cyanine-type photosensitizer. ACS Nano. 2011;5(7):5594-5607.
22. Agarwal A, Mackey MA, EI-sayed MA, Bellamkonda RV. Remote triggered release of doxorubicin in tumors by synergistic application of thermosensitive liposomes and gold nanorods. ACS Nano. 2011;5(6):4919-4926.

23. Cern A, Golbraikh A, Sedykh A, Tropsha A, Barenholz Y, Goldblum A. Quantitative structure property relationship modeling of remote liposome loading of drugs. J Control Release. 2012;160(2):147-157.

24. Tagami T, May JP, Ernsting MJ, Li SD. A thermosensitive liposome prepared with a $\mathrm{Cu}^{2+}$ gradient demonstrates improved pharmacokinetics, drug delivery and antitumor efficacy. $J$ Control Release. 2012;161(1):142-149.

25. Haran G, Cohen R, Bar LK, Barenholz Y. Transmembrane ammonium sulfate gradients in liposomes produce efficient and stable entrapment of amphipathic weak bases. Biochim Biophys Acta. 1993;1151(2):201-215.

26. Han HD, Byeon Y, Jeon HN, Shin BC. Enhanced localization of anticancer drug in tumor tissue using polyethylenimine-conjugated cationic liposomes. Nanoscale Res Lett. 2014;9(1):209.

27. Liao ZX, Chuang EY, Lin CC, et al. An AS1411 aptamer-conjugated liposomal system containing a bubble-generating agent for tumorspecific chemotherapy that overcomes multidrug resistance. $J$ Control Release. 2015;208:42-51.

28. Lim HJ, Oh CH. Indocyanine green-based photodynamic therapy with $785 \mathrm{~nm}$ light emitting diode for oral squamous cancer cells. Photodiagnosis Photodyn Ther. 2011;8(4):337-342.

29. Tomayko MM, Reynolds CP. Determination of subcutaneous tumor size in athymic (nude) mice. Cancer Chemother Pharmacol. 1989;24(3):148-154.

30. Yu M, Guo F, Tan FP, Li N. Dual-targeting nanocarrier system based on thermosensitive liposomes and gold nanorods for cancer thermochemotherapy. J Control Release. 2015;215:91-100.

31. Guo M, Mao HJ, Li YL, et al. Dual imaging-guided photothermal/photodynamic therapy using micelles. Biomaterials. 2014; 35(16):4656-4666.

32. Altinoglu EI, Russin TJ, Kaiser JM, et al. Near-infrared emitting fluorophore-doped calcium phosphate nanoparticles for in vivo imaging of human breast cancer. ACS Nano. 2008;2(10):2075-2084.

33. Cabral H, Matsumoto Y, Mizuno K, et al. Accumulation of sub- $100 \mathrm{~nm}$ polymeric micelles in poorly permeable tumours depends on size. Nat Nanotechnol. 2011;6(12):815-823.

34. Li L, Gao FP, Tang HB, et al. Self-assembled nanoparticles of cholesterol-conjugated carboxymethyl curdlan as a novel carrier of epirubicin. Nanotechnology. 2010;21(26):265601.

35. Kirchherr A, Briel A, Mader K. Stabilization of indocyanine green by encapsulation within micellar systems. Mol Pharm. 2009;6(2):480-491.

36. Jang B, Park JY, Tung CH, Kim IH, Choi Y. Gold nanorod-photosensitizer complex for near-infrared fluorescence imaging and photodynamic/ photothermal therapy in vivo. ACS Nano. 2011;5(2):1086-1094.

37. You J, Zhang G, Li C. Exceptionally high payload of doxorubicin in hollow gold nanospheres for near-infrared light-triggered drug release. ACS Nano. 2010;4(2):1033-1041.

38. Tagami T, Foltz W, Ernsting M, et al. MRI monitoring of intratumoral drug delivery and prediction of the therapeutic effect with a multifunctional thermosensitive liposome. Biomaterials. 2011; 32(27):6570-6578.

39. Drummond DC, Meyer O, Hong K, Kirpotin DB, Papahadjopoulos D. Optimizing liposomes for delivery of chemotherapeutic agents to solid tumors. Pharmacol Rev. 1999;51(4):691-743.

40. Boya P, Kroemer G. Lysosomal membrane permeabilization in cell death. Oncogene. 2008;27(50):6434-6451.

41. Zhu Q, Jia LX, Gao ZF, et al. A tumor environment responsive doxorubicin-loaded nanoparticle for targeted cancer therapy. Mol Pharm. 2014;11(10):3269-3278. 
International Journal of Nanomedicine

Dovepress

\section{Publish your work in this journal}

The International Journal of Nanomedicine is an international, peerreviewed journal focusing on the application of nanotechnology in diagnostics, therapeutics, and drug delivery systems throughou the biomedical field. This journal is indexed on PubMed Central, MedLine, CAS, SciSearch ${ }^{\circledR}$, Current Contents ${ }^{\circledR} /$ Clinical Medicine,
Journal Citation Reports/Science Edition, EMBase, Scopus and the Elsevier Bibliographic databases. The manuscript management system is completely online and includes a very quick and fair peer-review system, which is all easy to use. Visit http://www.dovepress.com/ testimonials.php to read real quotes from published authors.

Submit your manuscript here: http://www.dovepress.com/international-journal-of-nanomedicine-journal 